\title{
Les deux pactes mondiaux sur les réfugiés et les migrations : forces et faiblesses d'une nouvelle coopération internationale
}

The Two Global Compacts on Migration and Refugees: Strengths and Weaknesses of a New International Cooperation

Los dos pactos globales sobre los refugiados y sobre migración: fortalezas y

debilidades de la nueva cooperación internacional

Thibaut Fleury Graff

\section{(2) OpenEdition}

\section{Journals}

Édition électronique

URL : https://journals.openedition.org/remi/12112

DOI : $10.4000 /$ remi. 12112

ISSN : $1777-5418$

Éditeur

Université de Poitiers

Édition imprimée

Date de publication : 1 décembre 2018

Pagination : 223-230

ISBN : 979-10-90426-63-4

ISSN : 0765-0752

Référence électronique

Thibaut Fleury Graff, «Les deux pactes mondiaux sur les réfugiés et les migrations : forces et

faiblesses d'une nouvelle coopération internationale », Revue européenne des migrations internationales [En ligne], vol. 34 - n4 | 2018, mis en ligne le 01 janvier 2021, consulté le 14 avril 2022. URL : http:// journals.openedition.org/remi/12112; DOI : https://doi.org/10.4000/remi.12112 


\section{Chronique juridique}

\section{Les deux pactes mondiaux sur les réfugiés et les migrations : forces et faiblesses d'une nouvelle coopération internationale}

\section{Thibaut Fleury Graff ${ }^{1}$}

Les migrations internationales se sont multipliées ces dernières années, à un rythme qui n'avait pas été aussi soutenu depuis la fin de la Seconde Guerre mondiale (Edwards, 2017). Elles ont occupé le devant de la scène politique et médiatique dans de nombreux États - y compris, et peut-être surtout, dans ceux qui n'étaient que modestement concernés par ces migrations. L'un des exemples récents de cette attention portée à ces questions est celui des réactions provoquées par l'adoption, en décembre 2018, du « Pacte mondial pour des migrations sûres, ordonnées et régulières " (ci-après " $P M$ "). La signature de ce Pacte a en effet donné lieu à un " déchaînement de désinformation " de la part des partis populistes, certains allant jusqu'à évoquer un " pacte avec le diable ne visant qu'à transformer le monde en grand squat $\|^{2}$ ! Cette défiance n'a pas été sans conséquence, puisque quelques États - à l'image des États-Unis d'Amérique, de la Hongrie ou de la Pologne - ont voté contre le texte ${ }^{3}$. Ils sont cependant (très) loin d'être majoritaires, et l'adoption du Pacte, en dépit de ces quelques controverses juridiquement infondées, doit être vue comme un succès, de même que l'adoption, quelques jours auparavant, du "Pacte mondial sur les réfugiés" (ci-après « $P R$ ») - qui, pour sa part, a beaucoup moins fait parler de lui.

Ces deux Pactes constituent juridiquement des résolutions de I'Assemblée générale des Nations unies ${ }^{4}$. Ils sont le résultat d'un processus de négociations

\footnotetext{
1 Professeur de droit public, IDPSP, Université Rennes 1, Faculté de droit, 9 rue Jean Macé, CS 54203, 35042 Rennes Cedex ; thibaut.fleury-graff@univ-rennes1.fr

2 Cf. Le Monde (10 décembre 2018) Manipulations autour du pacte mondial sur l'immigration de I'ONU.

3 Cf. ONU info (19 décembre 2018) Migrations ; I'Assemblée générale des Nations unies endosse le Pacte de Marrakech. Les listes des États ayant voté pour ou contre le texte, ou s'étant abstenus, y est indiquée.

4 "Pacte mondial pour des migrations sûres, ordonnées et régulières ", Résolution A/ RES/73/195 adoptée par l'Assemblée générale des Nations unies le 19 décembre 2018. "Haut-Commissariat des Nations unies pour les Réfugiés ", Résolution A/RES/73/151 adoptée par l'Assemblée générale des Nations unies le 18 décembre 2018. Le texte du Pacte, facilement accessible en ligne, se trouve, dans sa version officielle, annexé au rapport du HCR qu'endosse la résolution 73/151.
} 
de près de deux ans, qui aura associé tant les États que diverses organisations internationales et acteurs de la société civile ${ }^{5}$. Ils trouvent leur principale origine dans le "Programme de développement durable à I'horizon 2030 ", adopté par les États membres de I'ONU en septembre $2015^{6}$, et dont le dixième objectif prévoit l'adoption de mesures destinées à " faciliter la migration et la mobilité de façon ordonnée, sûre, régulière et responsable, notamment par la mise en œuvre de politiques migratoires planifiées et bien gérées ". Un an plus tard, la fameuse "Déclaration de New York ", adoptée à l'unanimité et s'appuyant notamment sur le rapport In Safety and Dignity du Secrétaire général de I'ONU Ban Ki-Moon? , va concrétiser le projet de négociation et d'adoption de deux textes destinés à être les premiers instruments internationaux universels $\mathrm{d}^{\prime}$ encadrement de la coopération interétatique en la matière ${ }^{8}$. Rappelant que " depuis que le monde est monde, les hommes se déplacent soit pour rechercher de nouvelles perspectives et de nouveaux débouchés économiques, soit pour échapper à des conflits armés, à la pauvreté, à l'insécurité alimentaire, à la persécution, au terrorisme ou à des violations des droits de l'homme, soit enfin en réaction aux effets négatifs des changements climatiques, des catastrophes naturelles [...] ou d'autres facteurs environnementaux ", la Déclaration contient un certain nombre d'" engagements " concernant les réfugiés et les migrants, concrétisés et précisés dans les deux Pactes adoptés fin 2018. Ceux-ci constituent, en cela, un encadrement inédit de la coopération internationale en la matière - encadrement qui demeure toutefois non contraignant et dont l'avenir dépend donc largement de la bonne volonté des États.

\section{Un encadrement inédit de la coopération internationale concernant les migrants et les réfugiés}

La Convention de Genève relative au statut des réfugiés exprimait en 1951 "le vœu que tous les États reconnaissent le caractère social et humanitaire du problème des réfugiés " et qu'ils " fassent tout ce qui est en leur pouvoir pour éviter que ce problème ne devienne une cause de tension entre États " ${ }^{9}$. Depuis lors pourtant, aucun texte international d'envergure n'était venu initier de coopération internationale en matière de migrations - que cette migration fut celle de personnes entrant, ou non, dans le champ d'application de la Convention de 1951. Seuls quelques accords temporaires et spécifiques ont été adoptés au fil du temps pour encadrer la coopération interétatique lors de crises pour-

\footnotetext{
5 Sur le processus de négociation, voir Klein Solomon et Sheldon (2019: 3). Cette revue a publié un dossier spécial sur les Pactes, dont les articles sont largement cités dans les lignes qui suivent, et disponibles à I'adresse : https://academic.oup.com/ijrl

6 « Transformer notre monde : le programme de développement durable à I'horizon 2030 ", Résolution A/RES/70/1 adoptée par l'Assemblée générale des Nations unies le 25 septembre 2015.

7 "In safety and dignity: addressing large movements of refugees and migrants ", Report of the Secretary General, Résolution A/70/59 du 21 avril 2016.

8 " Déclaration de New York pour les réfugiés et les migrants ", Résolution A/RES/71/1 adoptée par l'Assemblée générale des Nations unies le 19 septembre 2016.

9 Préambule de la Convention relative au statut des réfugiés, signée à Genève le 28 juillet 1951.
} 
voyeuses de migrations (Robinson, 2004) ${ }^{10}$. Les deux pactes de 2018 remédient à ce quasi-mutisme de la communauté internationale ${ }^{11}$, en rappelant tout $\mathrm{d}^{\prime}$ abord les obligations existantes des États concernant les réfugiés et les migrants, puis en fixant un certain nombre de principes et objectifs concernant chacune de ces deux catégories.

Les deux pactes sont en effet, et dès leurs premières lignes, très clairs sur l'importance primordiale des instruments internationaux existants en matière de droits de I'homme, dont ils rappellent I'applicabilité aux migrants, quels qu'ils soient. "Le Pacte mondial est guidé par les instruments internationaux pertinents relatifs aux droits de I'homme [...] » rappellent ainsi les États signataires dans le point 5 du PR, quand le PM liste, dès son deuxième point, une dizaine d'instruments internationaux en matière de droits de I'homme sur lesquels il " s'appuie ". Les États y ajoutent en outre qu' " en appliquant le Pacte mondial, [ils veillent] au respect, à la protection et à la réalisation des droits de I'homme de tous les migrants, quel que soit leur statut migratoire, à tous les stades de la migration ". Ils y " réaffirment " en outre leur " attachement à l'élimination de toutes les formes de discrimination, dont le racisme, la xénophobie et l'intolérance, à l'endroit des migrants et de leur famille " (point 15f). De ce point de vue, les deux Pactes ne sont certainement pas novateurs, mais ont le mérite de constituer un utile rappel des obligations des États à l'égard de tout être humain - migrant ou non, en situation régulière ou non. Ces nouveaux instruments sont, par contre, plus originaux et ambitieux dans les principes directeurs et objectifs qu'ils fixent concernant les migrants en général et les réfugiés en particulier.

\section{Principes et objectifs du Pacte mondial pour des migrations sûres, ordonnées et régulières}

Le PM constitue le premier cadre de coopération globale en matière migratoire. II a ainsi vocation à régir la question dans son ensemble, quelles que soient les causes de migrations - on notera d'ailleurs certaines références, plus ou moins explicites, aux migrations climatiques ${ }^{12}-$ et en préconisant assez clairement une approche favorable à celles-ci. Le Pacte repose en effet sur l'idée que les migrations sont un phénomène inhérent à l'espèce humaine, et qu'elles sont "facteurs de prospérité, d'innovation et de développement durable " et qu'une meilleure gouvernance " peut permettre d'optimiser ces effets positifs " (point 8). L'objectif général du PM est ainsi selon F. Crépeau de "faciliter la mobilité humaine " (Crépeau, 2019), en assurant un meilleur accueil et une meilleure intégration des migrants, mais également un retour de ceux-ci dans leur pays d'origine dans de meilleures conditions (objectif 21). Plus précisément, le PM s'articule autour de dix principes directeurs, que l'on peut diviser en deux catégories principales.

10 Ainsi le Comprehensive Plan of Action adopté en 1989 pour encadrer la réinstallation des réfugiés indochinois, voir Robinson (2004).

11 II ne faut pas négliger en effet, outre les exemples cités en note précédente, les nombreux accords conclus entre le HCR et certains États pour la réinstallation ou la relocalisation des réfugiés, ou les quelques initiatives internationales concernant plus largement la question des migrations, tels que les « Dialogues de haut niveau sur les migrations internationales et le développement " organisés en 2006 et 2013 (https:// www.un.org/fr/ga/68/meetings/migration//).

12 Voir notamment les objectifs 2, 5 et 21 et leur interprétation par Kälin (2019). 
La première regroupe ceux des principes qui ont trait aux modalités de l'action de la communauté internationale : il est affirmé à ce titre que la coopération internationale est nécessaire, aucun État ne pouvant " seul, faire face aux migrations " (principe b), même si la souveraineté étatique, notamment concernant le droit d'entrée et de séjour sur le territoire de l'État, doit être préservée (principe c). En outre, cette coopération doit mobiliser «l'ensemble des pouvoirs publics " (principe i) et de la société (principe j). La migration est en effet conçue comme " un phénomène multidimensionnel " qui ne peut être traité ni par un " seul secteur de gouvernement " ni par un seul acteur : le PM appelle à ce que soit instituée une véritable gouvernance des migrations associant " les diasporas, les populations locales, la société civile, les milieux universitaires, le secteur privé, les parlementaires, les syndicats, les institutions nationales de défense des droits de l'homme, les médias et les autres acteurs concernés " (principe j).

Les six autres principes directeurs donnent corps à cette coopération internationale et globale en précisant qu'elle doit donner " priorité à la dimension humaine ", en promouvant "le bien-être des migrants " (principe a), ce qui passe par la primauté du droit et des procédures régulières (principe d), le respect des droits de l'homme (principe f), et par une sensibilité particulière à la problématique "femmes-hommes " (principe g) et aux besoins des enfants (principe h). Enfin, cette coopération doit également être organisée de telle manière à favoriser le développement durable, le PM visant à " tirer parti du potentiel qu'offrent les migrations " (principe e).

Ces principes doivent guider la réalisation des vingt-trois objectifs assignés par le Pacte, dont certains ne font guère débat, quand d'autres sont davantage controversés. Ainsi, les objectifs relatifs à la collecte de données sur les migrations (objectif 1), à l'importance de munir tous les migrants de preuves d'identités légales (objectif 4), à la lutte contre le trafic d'êtres humains (objectif 9) ou encore à la gestion "intégrée, sûre et coordonnée des frontières " (objectif 11) sont l'objet de peu de débats. II en va autrement, par exemple, de l'objectif consistant à " faire en sorte que les filières de migration régulière soient accessibles et plus souples " (objectif 5), à " mettre en place des mécanismes de portabilité des droits de sécurité sociale et des avantages acquis " (objectif 22) ou, enfin, de "donner aux migrants [...] des moyens en faveur de la pleine intégration [...] " (objectif 16). Ces derniers objectifs seront sans aucun doute plus difficiles à atteindre que les premiers.

\section{Principes et objectifs du Pacte mondial sur les réfugiés}

Si le Pacte sur les migrations institue, comme on vient de le voir rapidement, un nouvel " écosystème " de la coopération internationale en matière de migrations, l'objectif du Pacte sur les réfugiés est très sensiblement différent (Aleinikoff, 2019). En effet, outre qu'il ne concerne que les "réfugiés ", le Pacte prend appui sur un terrain qui, à la différence du PM, n'est pas vierge normativement. La Convention de 1951 et son Protocole de 1967 sont des instruments très largement acceptés par les États de la communauté internationale. Le "réfugié » est ainsi une figure ancienne de la coopération internationale - ancienne, mais paradoxale : alors que les États se sont accordés de longue date sur la définition du réfugié, et que cette définition n'était pas négociable dans le cadre de l'adop- 
tion du $\mathrm{PR}^{13}$, elle s'inscrit dans une dimension statique de l'action étatique. La Convention de 1951 fait peser sur les États d'accueil un certain nombre d'obligations, indifférentes au contexte migratoire dans lequel elles s'inscrivent, et auxquelles ne répond aucune coopération interétatique, alors même que les réfugiés se répartissent de manière très inégale entre les pays. II n'existe ainsi dans la Convention aucun mécanisme de partage des réfugiés entre États plus ou moins sollicités. La réinstallation ou la relocalisation $n^{\prime} y$ sont pas mentionnées. C'est à cette situation que le Pacte sur les réfugiés tente de remédier.

Selon les termes mêmes du Pacte, celui-ci a donc notamment pour principe directeur, outre le respect des droits de l'homme et de la souveraineté étatique, " d'opérationnaliser les principes de partage de la charge et des responsabilités pour mieux protéger et assister les réfugiés, et soutenir les pays et communautés d'accueil " (point 5). Ses principaux objectifs, énumérés au point 7, sont ainsi d'« i) alléger la pression sur les pays d'accueil ", de "ii) renforcer l'autonomie des réfugiés ", d' "(iii) élargir l'accès aux solutions dans des pays tiers" et, enfin, de " iv) favoriser les conditions d'un retour dans les pays d'origine en sécurité et dans la dignité ". Pour ce faire, le Pacte s'appuie, en particulier, sur deux instruments : le "Cadre d'action global pour les réfugiés ", annexé à la Déclaration de New York et qu'il reprend, destiné à être mis en place " dans chaque situation donnant lieu à un déplacement massif de réfugiés " et comportant des mesures relatives à l'accueil et à l'admission des personnes déplacées (enregistrement, éligibilité à la qualité de réfugié, respect des droits de l'homme dans les camps, etc.), à la satisfaction des besoins immédiats et à plus long terme des réfugiés et des pays d'accueil, et à la définition de solutions durables (rapatriement ou réinstallation notamment) ${ }^{14}$. Mais le Pacte repose également sur un autre mécanisme : I' « arrangement pour le partage de la charge et des responsabilités ". II s'agit d'un arrangement mondial, qui prend la forme d'un Forum sur les réfugiés destiné à réunir les États tous les quatre ans afin d'échanger leurs bonnes pratiques en la matière (points 17 à 19 PR). Cet arrangement connaît également des déclinaisons régionales et nationales : le Pacte invite les États d'accueil ou groupes d'États à élaborer, avec l'appui du HCR ainsi que des autres États et parties prenantes, des plans stratégiques faisant mention de leurs besoins en matière de financements, de réinstallation ou de rapatriement. Ils peuvent pour cela faire appel à une " plateforme d'appui " organisée par le HCR.

En outre, au-delà de ces considérations assez techniques, le Pacte insiste sur I'importance de mettre en place " des mécanismes efficaces " alternatifs aux camps de réfugiés (point 54 ) ce qui passe notamment par une meilleure intégration de ceux-ci : le Pacte met en avant la nécessité de permettre aux réfugiés d'accéder à l'éducation, à l'emploi, à la santé, au logement, à l'alimentation, etc. (points 64 et s., intitulés "Satisfaire les besoins et soutenir les communautés").

À la différence du PM, le PR est ainsi tourné vers une logique (un peu) plus technique, visant à la fois, grâce au plan d'action global, à gérer plus effica-

13 Le pacte énonce clairement, dès son point 5, qu'il " repose sur le régime international de protection des réfugiés [...] au cœur duquel se trouvent la Convention de 1951 et son protocole de 1967 [...]" ".

14 Points 4 à 16 du Plan d'action global, annexé à la Déclaration de New York de 2016. 
cement l'arrivée massive de réfugiés dans un État et à favoriser, grâce à ses nombreux objectifs, l'intégration des réfugiés reconnus comme tels dans leur pays d'accueil. S'il s'agit sans conteste, en ce sens, du " texte le plus important depuis 1951 " en matière de réfugiés (Türk, 2019), il n'en demeure pas moins qu'il s'agit d'un texte non contraignant, dont l'efficacité est dès lors soumise à la bonne volonté des États et des nombreuses autres parties prenantes qu'il mentionne. Un constat auquel le PM n'échappe pas.

\section{Un encadrement non contraignant soumis à la bonne volonté des Etats et autres parties prenantes}

Les " deux Pactes " peuvent s'analyser comme " une combinaison de plusieurs accords et compromis, entre différents acteurs et à propos de problématiques diverses " (Gammeltoft-Hansen, 2019). Ils sont en effet, et avant tout, des textes programmatiques, invitant les États, mais aussi, et très largement, la société civile - ONG, associations, communautés de migrants, communautés d'accueils, syndicats, entreprises, acteurs religieux, etc. - à s'emparer de la question des migrations et des réfugiés, et de conclure entre eux de nouveaux accords mettant en œuvre les objectifs fixés par les Pactes. En outre, à la diversité des acteurs cités ne répond pas une architecture précise et soignée des rôles de ces derniers. Les Pactes sont pleins d'expression laissant une très large part à l'interprétation, tant quant à la nécessité pour un acteur d'agir ou non, que quant à la définition de son rôle lui-même : les parties prenantes doivent agir "si nécessaire ", "lorsque l'État le juge nécessaire ", ou " selon le contexte ", et doivent le faire "sans préjudice " des rôles des uns et des autres - ces rôles demeurant définis en des termes excessivement généraux : il s'agit de " mettre en place des structures et des mécanismes appropriés ", $d^{\prime}$ ' explorer les mesures politiques et les arrangements pour l'élimination des risques " ou encore "d'explorer les meilleurs moyens d'inclure les réfugiés et les membres des communautés d'accueil ". Cette indécision, faisant la part belle à des processus de consultations et de négociations, a fait dire à certains que les Pactes constituaient un "rêve de bureaucrate " (Hathaway, 2019). II s'agit, en outre, d'instruments qui laissent intouchée la souveraineté de l'État. Non seulement parce qu'ils ne sont pas contraignants - les deux Pactes le rappellent très expressément ${ }^{15}$ - mais également parce qu'ils rappellent tout également que l'État demeure seul compétent pour décider qui, et à quelles conditions, peut entrer et séjourner sur son territoire. Le PM " réaffirme " ainsi " le droit souverain des États de définir leurs politiques migratoires nationales et leur droit de gérer les migrations relevant de leur compétence, dans le respect du droit international ${ }^{16}$.

Ces critiques sont assurément légitimes. Les tensions générées au sein de I'Union européenne par l'augmentation des migrations et les difficultés éprouvées par les États membres pour mettre en place des solutions équitables de gestion de celles-ci témoignent de la quasi-impossibilité, même grâce à

15 Point 4 du PR : «Le Pacte mondial n'est pas juridiquement contraignant ». Point 7 du PM " Le présent pacte mondial établit un cadre de coopération juridiquement non contraignant".

16 Point 15c) du PM. Voir aussi le point 33 du PR. 
des textes contraignants, de mettre en place des politiques respectueuses des principes et objectifs énumérés par les Pactes (Lantero et al., 2019). Pour autant, deux considérations au moins permettent de nuancer les critiques adressées à ces nouveaux instruments universels. Tout d'abord, dans le contexte actuel de montée des populismes et de défiance à l'égard du multilatéralisme, l'adoption des deux Pactes est, en elle-même, une bonne nouvelle, et l'on voit à vrai dire difficilement comment des textes plus ambitieux auraient pu voir le jour. L'affirmation d'une volonté de coopérer en la matière est déjà, en elle-même, une avancée. Ensuite, et peut-être surtout, ces Pactes ont, en dépit de (ou grâce à) leur caractère non contraignant, déjà produit certains effets, en particulier sur le continent africain, où de nouvelles législations favorables à l'inclusion des réfugiés dans les sociétés d'accueil ont vu le jour ${ }^{17}$. L'incitation vaut peut-être parfois mieux que la contrainte, et les Pactes sont, de ce point de vue, assurément un succès.

\section{Références bibliographiques}

Aleinikoff Alexander T (2019) The unfinished work of the global compact on refugees, International Journal of Refugee Law, [online]. URL: https://academic. oup.com/ijrl/advance-article/doi/10.1093/ijrl/eey057/5258096

Crépeau François (2019) Towards a mobile and diverse world: "facilitating mobility" as a central objective of the global compact on migration, International Journal of Refugee Law, [online]. URL: https://academic.oup.com/ijrl/advancearticle/doi/10.1093/ijrl/eey054/5250652

Edwards Adrian (2017) Le nombre de personnes déplacées atteint son plus haut niveau depuis des décennies, Genève, UNHCR, [en ligne]. URL : https:// www.unhcr.org/fr/news/stories/2017/6/5943f3eca/nombre-personnes-deplaceesatteint-haut-niveau-decennies.html

Fleury Graff Thibaut et Marie Alexis (2019) Droit de I'asile, Paris, PUF, 346 p.

Gammeltoft-Hansen Thomas (2019) The normative impact of the global compact on refugees, International Journal of Refugee Law, [online]. URL: https:// academic.oup.com/ijrl/advance-article/doi/10.1093/ijrl/eey061/5310459

Hathaway James C. (2019)The global cop-out on Refugees, International Journal of Refugee Law, [online]. URL: https://academic.oup.com/ijrl/advance-article/ doi/10.1093/ijrl/eey062/5310192

Kälin Walter (2019) The global compact on migration: a ray of hope for disaster-displaced persons, International Journal of Refugee Law, [online]. URL: https://academic.oup.com/ijrl/article/30/4/664/5248396

Klein Solomon Michele and Sheldon Suzanne (2019) The global compact for migration: from the sustainable development goals to a comprehensive agreement on safe, orderly and regular migration, International Journal of Refugee Law, [online]. URL: https://academic.oup.com/ijrl/advance-article/ doi/10.1093/ijrl/eey065/5352615

17 Voir les exemples cités parTürk (2019: 4-5). 
Lantero Catherine, Marie Alexis et Moulier Isabelle (Dirs.) (à paraître) Le droit $d^{\prime}$ asile en situation d'afflux massif : tensions et ambiguittés, Paris, Pedone.

Robinson W. Courtland (2004) The comprehensive plan of action for Indochinese refugees, 1989-1997: sharing the burden and passing the buck, Journal of refugees studies, 17 (3), pp. 319-333.

Türk Volker (2019) The promise and potential of the global compact on refugees, International Journal of Refugee Law, [online]. URL: https://academic.oup.com/ ijrl/advance-article/doi/10.1093/ijrl/eey068/5310191 